\title{
LARGE-TIME BEHAVIOR \\ OF DETERMINISTIC PARTICLE APPROXIMATIONS TO THE NAVIER-STOKES EQUATIONS
}

\author{
GEORGES-HENRI COTTET
}

\begin{abstract}
We prove that for a class of deterministic vortex methods for the Navier-Stokes equations in two dimensions, the numerical solution decays for large time with the same rate as the exact solution. We substantiate our result with numerical experiments and with a remark concerning the problem of reinitialization of a distribution of particles.
\end{abstract}

\section{INTRODUCTION}

Starting from the original particle methods for purely convective problems, deterministic particle methods have been designed to handle diffusion perturbations of these problems. The diffusion is dealt with by modifying the weights of the particles, while these particles still move according to the convection part of the equation. More precisely, given the vorticity formulation of the two-dimensional Navier-Stokes equations in the whole space:

$$
\begin{aligned}
\frac{\partial \omega}{\partial t}+\nabla(u \omega)-\nu \Delta \omega & =0, \\
\omega(\cdot, 0) & =\omega_{0}, \\
\operatorname{div} u & =0, \\
\operatorname{curl} u & =\omega, \\
|u| & \stackrel{\infty}{\rightarrow},
\end{aligned}
$$

the idea is to approximate the vorticity field by a set of particles:

$$
\omega(x, t) \simeq \omega^{h}(x, t)=\sum_{j} v_{j} \omega_{j}(t) \delta\left(x-x_{j}(t)\right) .
$$

In the above equation $v_{j}$ are the volumes of the particles, which remain constant due to the incompressibility of the flow, and $\omega_{j}$ are the local values of the vorticity. The initial locations of the particles are typically along a Cartesian mesh, which gives

$$
x_{j}(0)=j h, \quad v_{j}=h^{2}, \quad \omega_{j}(0)=\omega_{0}(j h) \quad \text { for } j \in \mathbf{Z}^{2} .
$$

Received May 31, 1989; revised November 30, 1989.

1980 Mathematics Subject Classification (1985 Revision). Primary 65M99, 35Q10.

Research supported by ONR contracts N00014-86-K-0691 and N00014-88-K-0414, and NSF grant DMS88-11863. 
Then $x_{j}$ and $\omega_{j}$ evolve according to the laws:

$$
\dot{x}_{j}(t)=u\left(x_{j}(t), t\right), \quad \dot{\omega}_{j}(t)=\frac{\nu}{\varepsilon^{2}} \sum_{k} v_{k}\left(\omega_{k}-\omega_{j}\right) \Lambda_{\varepsilon}\left(x_{k}-x_{j}\right), \quad j \in \mathbf{Z}^{2} .
$$

A possible way to reconstruct the velocity field $u$ from the set of particles is to use the Biot-Savart law, involving eventually a regularization of the measure vorticity. As for the kernel $\Lambda_{\varepsilon}$, it is obtained through

$$
\Lambda_{\varepsilon}(x)=\varepsilon^{-2} \Lambda\left(\frac{x}{\varepsilon}\right)
$$

where $\Lambda$ is a symmetric kernel satisfying

$$
\Lambda(x)=\Lambda(|x|) ; \quad \int x_{i}^{2} \Lambda(x) d x=2, \quad i=1,2
$$

For the derivation of such approximations to the diffusion and their analysis in the linear case we refer to [5].

This paper is concerned with the problem of the convergence of such approximations to the Navier-Stokes equations for large time. The corresponding problem for finite time is analyzed elsewhere (see [3]). It is well known (see [6] for instance) that the solutions of the continuous problem (1.1)-(1.5) exhibit a decay for large time like $1 / \sqrt{\nu t}$ for the enstrophy. Our goal here is to prove that the same result holds for the above numerical method, independently of the discretization parameters. The result is also largely independent of the way the velocity is reconstructed. Together with [3], this proves the convergence of the deterministic particle methods for $t \in[0,+\infty)$.

For our asymptotic result to be valid, we need some assumptions, some of them essential, the others for technical simplicity. Let us list these assumptions.

H1. $\Lambda \geq 0$ and $\Lambda$ has compact support.

The positivity of the kernel is for us the simplest way to ensure the decay of the enstrophy. This excludes kernels which lead to approximation to the diffusion of order 4 or above. However, it is possible to formulate an alternative sufficient condition in terms of the Fourier transform of the kernel which allows the use of a wider class of kernels. For simplicity we omit this possibility here.

We also assume that the dispersion of the particles can be controlled in some sense uniformly in time. More precisely:

H2. There exist constants $c_{1}, c_{1}^{\prime} \geq 0$ and, for all time, a permutation $\Psi$ in $\mathbf{Z}^{2}$ such that

$$
\begin{aligned}
c_{1}^{\prime}|\Psi(k) \dot{h}-\Psi(j) h| & \leq\left|x_{k}(t)-x_{j}(t)\right| \\
& \leq c_{1}|\Psi(k) h-\Psi(j) h|, \quad t \geq 0, j, k \in \mathbf{Z}^{2} .
\end{aligned}
$$


The assumption $\mathrm{H} 2$ is essential in the proof. In $\S 2$ we will comment on the relevance of this assumption. Finally, we assume that

H3. $\omega_{0}$ has compact support.

This assumption significantly simplifies the proof. However, the fact that the size of this support does not appear in the constants involved in the estimates indicates that the assumption is not essential. Related to this assumption we also assume that the vorticity carried by particles initially outside a bounded domain surrounding this support is zero. If $K$ is the size of this domain, the diffusion scheme now reads as

$$
\dot{\omega}_{j}(t)= \begin{cases}\nu \varepsilon^{-2} \sum_{k} h^{2}\left(\omega_{k}-\omega_{j}\right) \Lambda_{\varepsilon}\left(x_{k}-x_{j}\right) & \text { if }|j h| \leq K \\ 0 & \text { otherwise }\end{cases}
$$

We assume that the disc of radius $K$ is invariant under the reorderings $\Psi$ introduced in H2. An interpretation of (1.6) is that $\omega=0$ is imposed as an artificial boundary condition at the ends of the computational domain. This must be contrasted with the usual way of dealing with these methods: in general, particles are only considered inside the computational domain, and the resulting artificial boundary condition is more likely (although not exactly) a homogeneous Neumann boundary condition on the vorticity. We will comment on the differences between both approaches when we come to the numerical experiments. We can now state our result.

Theorem. Under the assumptions $\mathrm{H} 1$ to $\mathrm{H} 3$, there exists a constant $C$ depending only on $c_{1}$ and $\left\|\omega_{0}\right\|_{1}=\sum_{k} h^{2}\left|\omega_{k}(0)\right|$ such that the solution of (1.6) satisfies

$$
\sum_{j} h^{2}\left|\omega_{j}(t)\right|^{2} \leq \frac{C}{\nu t}, \quad t \geq 1
$$

for $\varepsilon$ small enough and $h \leq \varepsilon^{1+s}, s>0$.

As mentioned earlier, our proof is very much conditioned by the assumption $\mathrm{H} 2$, although we believe that this is only for technical reasons. In general, this assumption does not seem to be satisfied for all time, unless something special is done to prevent particles from moving too far apart or too close together. In other words, for our result to be a real long-time result, we need a reinitialization process which is both consistent and enstrophy-decreasing. The following section describes such an algorithm.

In [4], the authors prove for a class of finite element methods error estimates that are valid for all times. In contrast, our result and proof are more in the spirit of [6]. The proof consists in using $\mathrm{H} 2$ in order to reduce our problem to one on a fixed grid, and then to translate M. E. Schoenbeck's Fourier techniques into a discrete Fourier analysis on this grid. As a matter of fact, it follows in particular from our analysis that the long-time decay (1.7) extends to any finite difference scheme, as long as the convective term $\nabla(u \omega)$ is treated in such a way that its contribution to the $L^{2}$ norm of the vorticity vanishes. 
An outline of the paper is as follows. In $\S 2$ we discuss the assumption $\mathrm{H} 2$ and we define a reinitialization algorithm which does not increase the enstrophy. We also give an intermediate result based on quadrature formulas. In $\S 3$ we prove our theorem. Section 4 is finally devoted to numerical illustrations of our result and some practical conclusions for the implementation of the method.

\section{TWO INTERMEDIATE RESULTS AND A REINITIALIZATION ALGORITHM WHICH DOES NOT INCREASE THE ENSTROPHY}

To begin with, let us discuss the assumption H2. Consider first the case where we enforce $\Psi \equiv \mathrm{Id}$. It is clear that the dispersion of the particles (that is, the maximum among the quantities $\left.\left|x_{k}(t)-x_{j}(t)\right| /|k h-j h|,|k h-j h| /\left|x_{k}(t)-x_{j}(t)\right|\right)$ is controlled by the derivatives of the velocity, in a way that is exponential in time. This obviously makes the assumption $\mathrm{H} 2$ hard to satisfy. Even if we assume a decay of the first derivatives of the velocity like $1 / \sqrt{(\nu t)}$ (which is not anyway a direct consequence of our result), this exponential growth of the dispersion can only be improved into a polynomial growth. Although the permutation $\Psi$ adds some flexibility (which in practice can be useful; see numerical examples in $\S 4$ ), it seems difficult to predict in general that $\mathrm{H} 2$ will be satisfied, unless something is done to prevent the growth in the dispersion. The most natural thing is to reinitialize the distribution of particles along a uniform mesh after a fixed amount of time independent of the numerical parameters. This in turn raises the problem of finding a reinitialization process which does not deteriorate the decay of the enstrophy. We describe such a method, which is inspired from recent works in Particle-In-Cell methods. The problem can be stated as follows: given a distribution of particles $\left(x_{p}, \omega_{p}\right)_{p}$, find a new distribution on the uniform mesh $\left(p h, \tilde{\omega}_{p}\right)$ consistent with the original distribution and such that

$$
\sum_{p} h^{2}\left|\tilde{\omega}_{p}\right|^{2} \leq \sum_{p} h^{2}\left|\omega_{p}\right|^{2}
$$

For this we need an intermediate grid with grid size $\eta \gg h$. Throughout this section, indices $j, k$ refer to this grid, while indices $p, q$ refer to the particles. Associated with the grid just introduced, we consider a symmetric basis function $\phi$, that is, a function such that

$$
\sum_{j} \phi\left(\frac{x_{j}-x}{\eta}\right)=1 \text { for all } x \in \mathbf{R}^{2}
$$

Following [1], we assign the vorticity on the grid by defining

$$
\beta_{j}=\sum_{p} h^{2} \phi\left(\frac{x_{j}-x_{p}}{\eta}\right), \quad \omega_{j}^{\prime}=\frac{1}{\beta_{j}} \sum_{p} h^{2} \omega_{p} \phi\left(\frac{x_{j}-x_{p}}{\eta}\right) .
$$


The coefficients $\beta_{j}$ are clearly an approximation of $\eta^{2}$, which makes the above assignment scheme consistent. Then we go back to the particles by setting

$$
\begin{gathered}
\beta_{j}^{\prime}=\sum_{p} h^{2} \phi\left(\frac{x_{j}-p h}{\eta}\right), \quad \gamma_{j}=\left(\frac{\beta_{j}}{\beta_{j}^{\prime}}\right)^{1 / 2}, \\
\tilde{\omega}_{p}=\sum_{j} \omega_{j}^{\prime} \gamma_{j} \phi\left(\frac{p h-x_{j}}{\eta}\right),
\end{gathered}
$$

which is also a consistent way of computing the value of the vorticity at the points $p h$. The above process introduces some diffusion, but certainly no oscillations: we can prove

Proposition. The reinitialization process defined by (2.2), (2.3) is stable in the following sense:

$$
\sum_{p} h^{2}\left|\tilde{\omega}_{p}\right|^{2} \leq \sum_{p} h^{2}\left|\omega_{p}\right|^{2}
$$

Proof. First we write, using (2.3),

$$
\left|\tilde{\omega}_{p}\right|^{2}=\sum_{j k} \omega_{j}^{\prime} \omega_{k}^{\prime} \gamma_{j} \gamma_{k} \phi\left(\frac{p h-x_{j}}{\eta}\right) \phi\left(\frac{p h-x_{k}}{\eta}\right) .
$$

Writing then $\left|\omega_{j}^{\prime} \omega_{k}^{\prime} \gamma_{j} \gamma_{k}\right| \leq \frac{1}{2}\left(\left|\gamma_{j} \omega_{j}^{\prime}\right|^{2}+\left|\gamma_{k} \omega_{k}^{\prime}\right|^{2}\right)$ yields

$$
\begin{aligned}
\left|\tilde{\omega}_{p}\right|^{2} & \leq \sum_{j}\left|\gamma_{j} \omega_{j}^{\prime}\right|^{2} \phi\left(\frac{p h-x_{j}}{\eta}\right) \sum_{k} \phi\left(\frac{p h-x_{k}}{\eta}\right) \\
& \leq \sum_{j}\left|\gamma_{j} \omega_{j}^{\prime}\right|^{2} \phi\left(\frac{p h-x_{j}}{\eta}\right),
\end{aligned}
$$

where for the last inequality we have used (2.1). Using now the definitions of $\beta_{j}, \beta_{j}^{\prime}, \gamma_{j}$, we obtain

$$
\sum_{p} h^{2}\left|\tilde{\omega}_{p}\right|^{2} \leq \sum_{j}\left|\omega_{j}^{\prime}\right|^{2} \gamma_{j}^{2} \beta_{j}^{\prime}=\sum_{j}\left|\omega_{j}^{\prime}\right|^{2} \beta_{j} .
$$

Next (2.2) gives

$$
\begin{aligned}
\sum_{j}\left|\omega_{j}^{\prime}\right|^{2} \beta_{j} & =\sum_{j} \frac{h^{4}}{\beta_{j}} \sum_{p q} \omega_{p} \omega_{q} \phi\left(\frac{x_{p}-x_{j}}{\eta}\right) \phi\left(\frac{x_{q}-x_{j}}{\eta}\right) \\
& \leq \sum_{p} h^{2}\left|\omega_{p}\right|^{2} \sum_{j} \frac{1}{\beta_{j}} \sum_{q} h^{2} \phi\left(\frac{x_{p}-x_{j}}{\eta}\right) \phi\left(\frac{x_{q}-x_{j}}{\eta}\right) .
\end{aligned}
$$

But from (2.2) we have

$$
\sum_{q} h^{2} \phi\left(\frac{x_{p}-x_{j}}{\eta}\right) \phi\left(\frac{x_{q}-x_{j}}{\eta}\right)=\beta_{j} \phi\left(\frac{x_{p}-x_{j}}{\eta}\right),
$$


and thus

$$
\sum_{j}\left|\omega_{j}^{\prime}\right|^{2} \beta_{j} \leq \sum_{p} h^{2}\left|\omega_{p}\right|^{2} \sum_{j} \phi\left(\frac{x_{p}-x_{j}}{\eta}\right)=\sum_{p} h^{2}\left|\omega_{p}\right|^{2},
$$

which along with (2.5) proves (2.4).

Let us now give an intermediate result based on quadrature estimates.

Lemma 1. For $j, l \in \mathbf{Z}^{2}, \varepsilon$ small enough, and $h \leq \varepsilon^{1+s}$, the following estimates hold:

$$
\begin{gathered}
\sum_{k} h^{2}\left(k_{i} h-j_{i} h\right)^{2} \Lambda_{\varepsilon}\left(c_{1}(k h-j h)\right) \geq C \varepsilon^{2}, \\
\varepsilon^{-2} \sum_{k} h^{2}\left|\sin \frac{h}{2}\left\langle k_{i}-j_{i}, l\right\rangle\right|^{2} \Lambda_{\varepsilon}\left(c_{1}(k h-j h)\right) \\
\geq C \begin{cases}|l|^{2} & \text { if }|l| \leq \varepsilon^{-1}, \\
\varepsilon^{-2} & \text { otherwise. }\end{cases}
\end{gathered}
$$

Proof. We first consider the continuous integral which is behind the discrete sum in the left-hand side of (2.6). We get immediately from the definition of $\Lambda_{\varepsilon}$

$$
\int\left(x_{i}-j_{i} h\right)^{2} \Lambda_{\varepsilon}\left(c_{1}(x-j h)\right) d x=2 c_{1}^{-4} \varepsilon^{2}, \quad j \in \mathbf{Z}^{2}, i=1,2 .
$$

As a result, the standard estimates for the midpoint quadrature rule give for $m \geq 2$ :

$$
\begin{aligned}
& \left|\sum_{k} h^{2}\left(k_{i} h-j_{i} h\right)^{2} \Lambda_{\varepsilon}\left(c_{1}(k h-j h)\right)-2 c_{1}^{-4} \varepsilon^{2}\right| \\
& \quad \leq C_{m} h^{m}\left[\varepsilon^{2}\left|\Lambda_{\varepsilon}\right|_{m, 1}+\varepsilon\left|\Lambda_{\varepsilon}\right|_{m-1,1}+\left|\Lambda_{\varepsilon}\right|_{m-2,1}\right] .
\end{aligned}
$$

Since $\left|\Lambda_{\varepsilon}\right|_{p, 1}=c_{p} \varepsilon^{-p}$, we have

$$
\left|\sum_{k} h^{2}\left(k_{i} h-j_{i} h\right)^{2} \Lambda_{\varepsilon}\left(c_{1}(k h-j h)\right)-2 c_{1}^{-4} \varepsilon^{2}\right| \leq C_{m} h^{m} \varepsilon^{-m}
$$

and (2.6) will be satisfied as soon as $\varepsilon$ is small enough and $h \ll \varepsilon$. For (2.7) we need to consider

$$
I(l)=\varepsilon^{-2} \int\left|\sin \frac{1}{2}\langle y-x, l\rangle\right|^{2} \Lambda_{\varepsilon}\left(c_{1}(y-x)\right) d y .
$$

If $\rho$ is the finite size of the support of $\Lambda$, the integral is only over $y$ such that $|y-x| \leq \rho \varepsilon$. Using the change of variables $y-x=\varepsilon z$, we obtain

$$
I(l)=\varepsilon^{-2} \int_{|z| \leq \rho}\left|\sin \frac{1}{2}\langle z, \varepsilon l\rangle\right|^{2} \Lambda\left(c_{1} z\right) d z .
$$


If we set $\varepsilon l=\alpha$, we have, for $|\alpha| \leq \pi / 4 \rho$ and $|z| \leq \rho,\left|\sin \frac{1}{2}\langle z, \varepsilon l\rangle\right| \geq$ $|\langle z, \alpha\rangle| / \pi$ and therefore

$$
I(l) \geq C \varepsilon^{-2} \int \Lambda\left(c_{1} z\right)\left(z_{1}^{2} \alpha_{1}^{2}+z_{2}^{2} \alpha_{2}^{2}+2 z_{1} z_{2} \alpha_{1} \alpha_{2}\right) d z,
$$

which in view of the properties of $\Lambda$ yields

$$
I(l) \geq 2 C c_{1}^{-4}|\alpha|^{2}=C^{\prime}|l|^{2} \varepsilon^{-2} \text { for } l \leq \pi / 4 \rho \varepsilon
$$

Next, for $|\alpha| \geq \pi /(4 \rho)$, we can rewrite

$$
\left|\sin \frac{1}{2}\langle z, \varepsilon l\rangle\right|=\frac{1}{2}(1-\cos \langle z, \alpha\rangle) .
$$

Therefore, on the one hand we get

$$
\lim _{|\alpha| \rightarrow \infty} \int \Lambda\left(c_{1} z\right)\left|\sin \frac{1}{2}\langle z, \alpha\rangle\right|^{2} d z=\frac{1}{2} \int \Lambda\left(c_{1} z\right) d z .
$$

On the other hand, the above integral clearly never takes the value 0 . As a result, we can write

$$
\int \Lambda\left(c_{1} z\right)\left|\sin \frac{1}{2}\langle z, \alpha\rangle\right|^{2} d z \geq C, \quad|\alpha| \geq \pi / 4,
$$

or, equivalently,

$$
I(l) \geq C \varepsilon^{-2} \text { for } l \geq \pi /(4 \rho \varepsilon) .
$$

Finally, the estimates (2.7) on the discrete sums can be deduced from their analogue (2.8) and (2.9) on the continuous integrals by applying the same quadrature argument as previously.

\section{Proof of THE THEOREM}

For the sake of simplicity in the notations we give the proof in the case $\Psi \equiv$ Id. The general case can be recovered through straightforward modifications. We first introduce the tools and notations needed in the proof. We rewrite the constant $K$ introduced in (1.6) as $K=\pi M$ and we assume that $\pi(M+1) / h-$ $1 / 2$ is an integer. We then set

$$
\alpha=\frac{h}{(M+1)}, \quad N=\frac{\pi(M+1)}{h}-\frac{1}{2} .
$$

Next we define the discrete Fourier coefficients:

$$
\hat{\omega}_{l}=\sum_{k} h^{2} \omega_{k} \exp (i \alpha\langle l, k\rangle), \quad l \in[-N, N]^{2} .
$$

For $j \in \mathbf{Z}^{2}$ we denote by $j^{\prime}$ the only integer such that $j^{\prime} h-j h \in 2 \pi(M+1) \mathbf{Z}^{2}$ and $\left|j^{\prime} h\right| \leq \pi(M+1)$. The following classical result gives the Parseval identity and sets up the rules for computing the inverse Fourier transform. 
Lemma 2. If $\omega_{j}=0$ for $|j h| \geq \pi M$, then we have

$$
\begin{gathered}
\omega_{j^{\prime}}=\frac{1}{\pi^{2}(M+1)^{2}} \sum_{|l| \leq N} \hat{\omega}_{l} \exp (-i \alpha\langle l, j\rangle), \\
\sum_{j} h^{2}\left|\omega_{j}\right|^{2}=\frac{1}{\pi^{2}(M+1)^{2}} \sum_{|l| \leq N}\left|\hat{\omega}_{l}\right|^{2} .
\end{gathered}
$$

Proof. Substituting (3.1) in the right-hand side of (3.2) gives

$$
\sum_{|l| \leq N} \hat{\omega}_{l} \exp (-i \alpha\langle l, j\rangle)=\sum_{k} \sum_{|l| \leq N} h^{2} \omega_{k} \exp (i \alpha\langle l, k-j\rangle) .
$$

If the components of $\alpha(k-j)$ are not multiples of $2 \pi$, then

$$
\begin{aligned}
& \sum_{|l| \leq N} h^{2} \omega_{k} \exp (i \alpha\langle l, k-j\rangle) \\
& \quad=\frac{\sin \frac{\alpha}{2}(N+1 / 2)\left(k_{1}-j_{1}\right)}{\sin \frac{\alpha}{2}\left(k_{1}-j_{1}\right)} \frac{\sin \frac{\alpha}{2}(N+1 / 2)\left(k_{2}-j_{2}\right)}{\sin \frac{\alpha}{2}\left(k_{2}-j_{2}\right)}=0,
\end{aligned}
$$

and, by the assumption on the support of $\omega$, the only $k$ not falling in this case and with $\omega_{k} \neq 0$ is $j^{\prime}$. As a result

$$
\sum_{|l| \leq N} \hat{\omega}_{l} \exp (-i \alpha\langle l, j\rangle)=(2 N-1)^{2} \omega_{j^{\prime}}
$$

which proves (3.2). (3.3) is proved in the same way.

In the sequel we will use the notations $\Omega=\pi^{-2}(M+1)^{-2}$ and $\lambda_{j k}=$ $\Lambda_{\varepsilon}\left(c_{1}(k h-j h)\right)$. The next step is to prove

Lemma 3. Under the assumption of Lemma 1 and $\mathrm{H} 1, \mathrm{H} 2$, we have for $\varepsilon$ small enough

$$
\begin{aligned}
& \sum_{k, j}\left(\omega_{k}-\omega_{j}\right)^{2} \Lambda_{\varepsilon}\left(x_{k}-x_{j}\right) \\
& \quad \geq \Omega^{2}(2 N-1)^{2} \sum_{l}\left|\hat{\omega}_{l}\right|^{2} \sum_{k}\left|\sin \frac{\alpha}{2}\langle k-j, l\rangle\right|^{2} \lambda_{j k} .
\end{aligned}
$$

Proof. We develop the left-hand side of (3.4) after substituting (3.2). First we observe that $\mathrm{H} 1$ implies that $\Lambda$ is a decreasing function of the modulus of the argument. Therefore, in view of $\mathrm{H} 2$,

$$
\Lambda_{\varepsilon}\left(x_{k}-x_{j}\right) \geq \lambda_{j k}
$$

and by (3.2),

$$
\begin{aligned}
\left(\omega_{k^{\prime}}-\right. & \left.\omega_{j^{\prime}}\right)^{2} \Lambda_{\varepsilon}\left(x_{k}-x_{j}\right) \\
\geq & \Omega^{2} \sum_{l, m} \hat{\omega}_{l} \hat{\omega}_{m} \exp (-i \alpha\langle j, l-m\rangle) \\
\quad & \quad\left\{\exp \left(-i \frac{\alpha}{2}\langle k-j, l-m\rangle\right)\right. \\
& \left.\quad \times \sin \frac{\alpha}{2}\langle k-j, l\rangle \sin \frac{\alpha}{2}\langle k-j, m\rangle \lambda_{k j}\right\} .
\end{aligned}
$$


Let $a_{j k}$ be the right-hand side of (3.5). We now introduce the following additional notations:

$$
\begin{aligned}
& S_{1}=\left\{j \in \mathbf{Z}^{2},|j h| \leq \pi(M+1)-h / 2=N h\right\}, \\
& S_{2}=\left\{j \in \mathbf{Z}^{2},|j h| \leq \pi(M+2)\right\} ;
\end{aligned}
$$

a little reflection will show that in the summation of the $a_{j k}$ for $j \in S_{1}$ and $k \in S_{2}$ there is actually no repetition of the corresponding indices $j^{\prime}, k^{\prime}$, and therefore we can use that sum to evaluate the left-hand side of (3.4). If $j \in S_{1}$, $k \in S_{2}$, then either $k \in S_{1}$, in which case $j^{\prime}=j, k^{\prime}=k$, or $k \in S_{2}-S_{1}$. In the latter case we have $\pi(M+1)<\left|k^{\prime} h\right| \leq \pi(M+3 / 2)$, and, by the assumption on the support of $\omega, \omega_{k^{\prime}}=0$. On the other hand, if $\left|j^{\prime} h\right|>\pi M$, we have $\omega_{j^{\prime}}=0$, while if $\left|j^{\prime} h\right| \leq \pi M$, then $|j h-k h|>\pi$. Now we use assumption $\mathrm{H} 2$ to deduce that $\left|x_{j}-x_{k}\right|>c_{1}^{\prime} \pi$ and therefore $\Lambda_{\varepsilon}\left(x_{j}-x_{k}\right)=0$ if $\varepsilon$ is small enough. To sum up, we have

$$
\begin{gathered}
j \in S_{1}, \quad k \in S_{1} \Rightarrow j^{\prime}=j, k^{\prime}=k \\
j \in S_{1}, \quad k \in S_{2}-S_{1} \Rightarrow\left(\omega_{k^{\prime}}-\omega_{j^{\prime}}\right) \Lambda_{\varepsilon}\left(x_{k}-x_{j}\right)=0 .
\end{gathered}
$$

Therefore,

$$
\sum_{j k}\left(\omega_{k}-\omega_{j}\right)^{2} \Lambda_{\varepsilon}\left(x_{k}-x_{j}\right) \geq \sum_{j \in S_{1}, k \in S_{2}} a_{j k}
$$

We next observe that if we denote by $A_{j k}^{l m}$ the term between braces in (3.5), then $\sum_{k \in S_{2}} A_{k j}^{l m}$ is independent of $j \in S_{1}$ if $\varepsilon$ is small enough. The reason is that $\lambda_{j k}=0$ if $c_{1}|j-k| h \geq 1$ and, in particular, for $\varepsilon$ small enough, as soon as $j \in S_{1}, k \notin S_{2}$. As a result,

$$
\sum_{k \in S_{2}} A_{j k}^{l m}=\sum_{k} A_{j k}^{l m}
$$

and the invariance under translation of $(k-j)$ proves our assertion. If we denote by $\beta_{l m}$ the quantity in (3.6), we therefore have

$$
\sum_{k, j}\left(\omega_{k}-\omega_{j}\right)^{2} \Lambda_{\varepsilon}\left(x_{k}-x_{j}\right) \geq \Omega^{2} \sum_{l, m} \hat{\omega}_{l} \hat{\omega}_{m} \beta_{l m} \sum_{j \in S_{1}} \exp (-i \alpha\langle j, l-m\rangle) .
$$

Finally, owing to the definition of $S_{1}$, the sum with respect to $j$ in the righthand side above is 0 , unless $l=m$. This yields

$$
\sum_{k, j}\left(\omega_{k}-\omega_{j}\right)^{2} \Lambda_{\varepsilon}\left(x_{k}-x_{j}\right) \geq \Omega^{2}(2 N-1)^{2} \sum_{l}\left|\hat{\omega}_{l}\right|^{2} \beta_{l l}
$$

which can be rewritten as (3.4). 
This allows us now to prove the following key result:

Lemma 4. Under the assumptions of Lemma 3 there exists a constant $C_{0}$ depending only on $c_{1}$ such that

$$
\frac{d}{d t} \sum_{l}\left|\hat{\omega}_{l}\right|^{2}+C_{0} \Omega \nu \sum_{l} \inf \left(l^{2}, M^{2} \varepsilon^{-2}\right)\left|\hat{\omega}_{l}\right|^{2} \leq 0 .
$$

Proof. Starting from (1.6), we observe that for all indices $j$ we have

$$
\dot{\omega}_{j} \omega_{j}=\frac{\nu}{\varepsilon^{2}} \sum_{k}\left(\omega_{k}-\omega_{j}\right) \omega_{j} \Lambda_{\varepsilon}\left(x_{k}-r\right) .
$$

Therefore, (3.3) yields

$$
\begin{aligned}
\frac{\Omega}{2} \frac{d}{d t} \sum_{l} h^{2}\left|\hat{\omega}_{l}\right|^{2} & =\frac{1}{2} \frac{d}{d t} \sum_{j} h^{2}\left|\omega_{j}\right|^{2} \\
& =\frac{\nu}{\varepsilon^{2}} \sum_{k, j} h^{4}\left(\omega_{k}-\omega_{j}\right) \omega_{j} \Lambda_{\varepsilon}\left(x_{k}-x_{j}\right) \\
& =-\frac{\nu}{2 \varepsilon^{2}} \sum_{k, j} h^{4}\left(\omega_{k}-\omega_{j}\right)^{2} \Lambda_{\varepsilon}\left(x_{k}-x_{j}\right),
\end{aligned}
$$

where for the last equality we have used the symmetry of the kernel $\Lambda_{\varepsilon}$. The above right-hand side gives the rate of dissipation obtained in the numerical method. Our goal here is to relate it to the one corresponding to the continuous equations. In view of (3.4) we have to estimate for any fixed index $l$

$$
I=\sum_{k}\left|\sin \frac{\alpha}{2}\langle k-j, l\rangle\right|^{2} \lambda_{j k}
$$

But it results directly from Lemma 1, estimate (2.7), that

$$
I \geq C h^{-2} \varepsilon^{2} \inf \left(l^{2} M^{-2}, \varepsilon^{-2}\right) \geq C h^{-2} \varepsilon^{2} \Omega \inf \left(l^{2}, M^{2} \varepsilon^{-2}\right) .
$$

It remains then to substitute the above inequality in (3.4), then in the right-hand side of (3.8), to get the desired inequality.

An interpretation of the above result is that the minimum scale $\varepsilon$ introduced in the numerical method results in a limitation of the rate of dissipation. However, this limitation does not affect the long-time behavior of the numerical solution, as will be seen now. We are in a situation where we can use the nice trick introduced in [6] to prove our theorem. We write $C_{1}=C_{0} / \Omega$ and we introduce a last notation:

$$
L=\left\{l \in \mathbf{Z}^{2},|l| \geq \sqrt{\frac{2}{\nu C_{1} t}}\right\}, \quad L^{\prime}=\mathbf{Z}^{2}-L .
$$

For $l \in L$ we have

$$
\inf \left(l^{2}, M^{2} \varepsilon^{-2}\right) \geq \inf \left(\frac{2}{C_{1} \nu t}, M^{2} \varepsilon^{-2}\right) \geq \frac{2}{C_{1} \nu t}
$$


if $t \geq 1$ and $\varepsilon$ is small enough. Therefore, we can write

$$
\begin{aligned}
\sum_{l}\left|\hat{\omega}_{l}\right|^{2} \inf \left(l^{2}, M^{2} \varepsilon^{-2}\right) & \geq \sum_{l \in L}\left|\hat{\omega}_{l}\right|^{2} \inf \left(l^{2}, M^{2} \varepsilon^{-2}\right) \geq \frac{2}{C_{1} \nu t} \sum_{l \in L}\left|\hat{\omega}_{l}\right|^{2} \\
& \geq \frac{2}{C_{1} \nu t}\left[\sum_{l}\left|\hat{\omega}_{l}\right|^{2}-\sum_{l \in L^{\prime}}\left|\hat{\omega}_{l}\right|^{2}\right] \\
& \geq \frac{2}{C_{1} \nu t}\left[\sum_{l}\left|\hat{\omega}_{l}\right|^{2}-\left|L^{\prime}\right| \max _{l}\left|\hat{\omega}_{l}\right|^{2}\right]
\end{aligned}
$$

Of course, $\left|L^{\prime}\right| \leq 2 / C_{1} \nu t$; on the other hand,

$$
\max _{l}\left|\hat{\omega}_{l}\right| \leq \sum_{k} h^{2}\left|\omega_{k}\right|
$$

In order to relate the above right-hand side to $\left\|\omega_{0}\right\|_{1}$, we define

$$
s_{j}= \begin{cases}1 & \text { if } \omega_{j}>0 \\ 0 & \text { if } \omega_{j}=0 \\ -1 & \text { if } \omega_{j}<0\end{cases}
$$

(1.6) implies that, for all $j$,

$$
\frac{d}{d t}\left|\omega_{j}\right|=\dot{\omega}_{j} s_{j}=\frac{\nu}{\varepsilon^{2}} \sum_{k} h^{2}\left(\omega_{k}-\omega_{j}\right) s_{j} \Lambda_{\varepsilon}\left(x_{k}-x_{j}\right) .
$$

Using again the symmetry of the kernel $\Lambda$, we then obtain

$$
\frac{d}{d t} \sum_{j} h^{2}\left|\omega_{j}\right|=\frac{\nu}{\varepsilon^{2}} \sum_{j k} h^{4}\left(\omega_{k}-\omega_{j}\right)\left(s_{j}-s_{k}\right) \Lambda_{\varepsilon}\left(x_{k}-x_{j}\right) \leq 0,
$$

which gives

$$
\sum_{j} h^{2}\left|\omega_{j}(t)\right| \leq \sum_{j} h^{2}\left|\omega_{j}(0)\right|=\left\|\omega_{0}\right\|_{1} .
$$

Hence, (3.9) now yields

$$
\sum_{l}|l|^{2}\left|\hat{\omega}_{l}\right|^{2} \geq \frac{2}{C_{1} \nu t} \sum_{l}\left|\hat{\omega}_{l}\right|^{2}-\frac{4}{\left(C_{1} \nu t\right)^{2}}\left\|\omega_{0}\right\|_{1}^{2} .
$$

If we set $y(t)=t^{2} \sum_{l}\left|\hat{\omega}_{l}(t)\right|^{2}$, we get from (3.7)

$$
\dot{y} \leq \frac{4}{C_{1} \nu}\left\|\omega_{0}\right\|_{1}^{2} \text {. }
$$

Therefore, we get for $t \geq 1$

$$
\sum_{l}\left|\hat{\omega}_{l}(t)\right|^{2} \leq C \frac{\Omega}{\nu t}\left\|\omega_{0}\right\|_{1}^{2}
$$

and using finally (3.3) proves our decay property (1.7). 


\section{NUMERICAL ILLUSTRATIONS}

We have performed experiments with the following initial vorticity:

$$
\omega_{0}(x)= \begin{cases}\left(1-|x|^{2}\right)^{3} & \text { if }|x| \leq 1 \\ 0 & \text { otherwise. }\end{cases}
$$

The particles were initially lying on a uniform mesh with $h=.15$ inside the disc of radius 1.5 , resulting in 316 particles. We have set $\varepsilon=2 h$, and have chosen $\Lambda(r)=16 / \pi\left(1+r^{2}\right)^{-3}$. For the time discretization of the various ordinary differential equations involved in the method, we have used a Runge-Kutta method of order 4 with $\Delta t=1$.

In the first experiment we have implemented the method in the usual way, which slightly differs from (1.6), that is, we have simply solved for all $j$ :

$$
\dot{\omega}_{j}(t)=\frac{\nu}{\varepsilon^{2}} \sum_{k} h^{2}\left(\omega_{k}-\omega_{j}\right) \Lambda_{\varepsilon}\left(x_{k}-x_{j}\right) .
$$

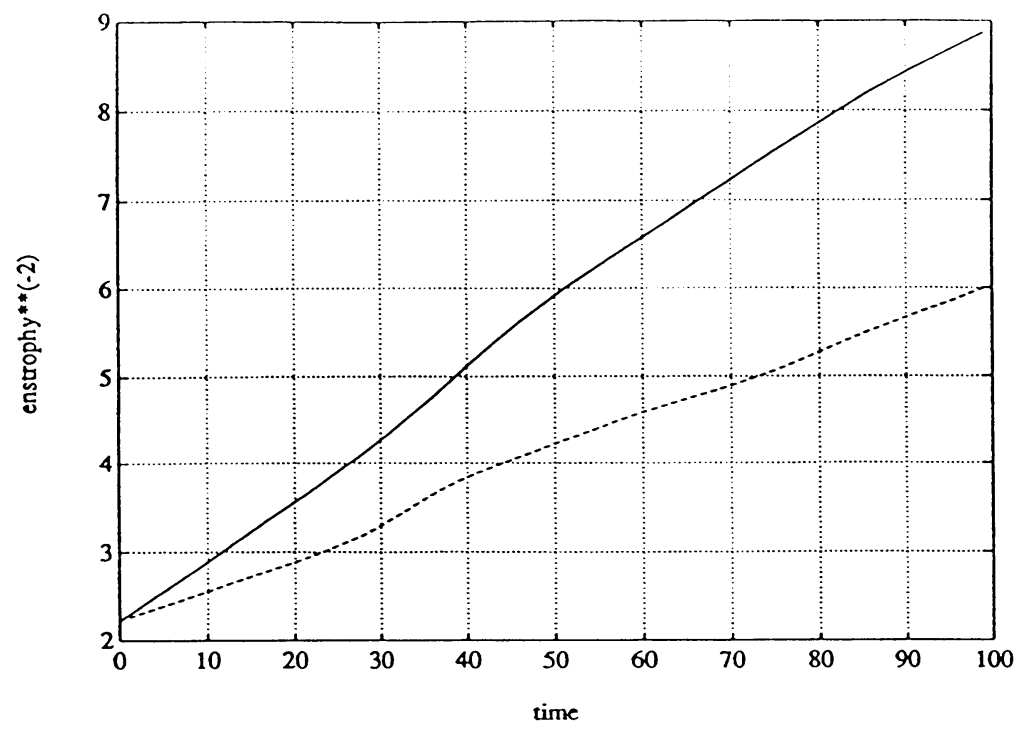

FIGURE 1

$\left(\sum_{j} h^{2}\left|\omega_{j}(t)\right|^{2}\right)^{-1}$ vs time for $\nu=10^{-3}$ (dotted line) and $\nu=$ $2 \cdot 10^{-3}$

Figure 1 represents the increase of $\left(\sum_{j} h^{2}\left|\omega_{j}(t)\right|^{2}\right)^{-1}$ as a function of time, for two small values of the viscosity, $\nu=10^{-3}$ and $\nu=2 \cdot 10^{-3}$. We observe that the linear growth predicted as a lower bound and the linear dependence on $\nu$ are quite well fitted, which is not surprising since, owing to the radial symmetry of the initial vorticity, the continuous equation behaves as a simple heat equation. We also must point out that no reinitialization has been needed. 


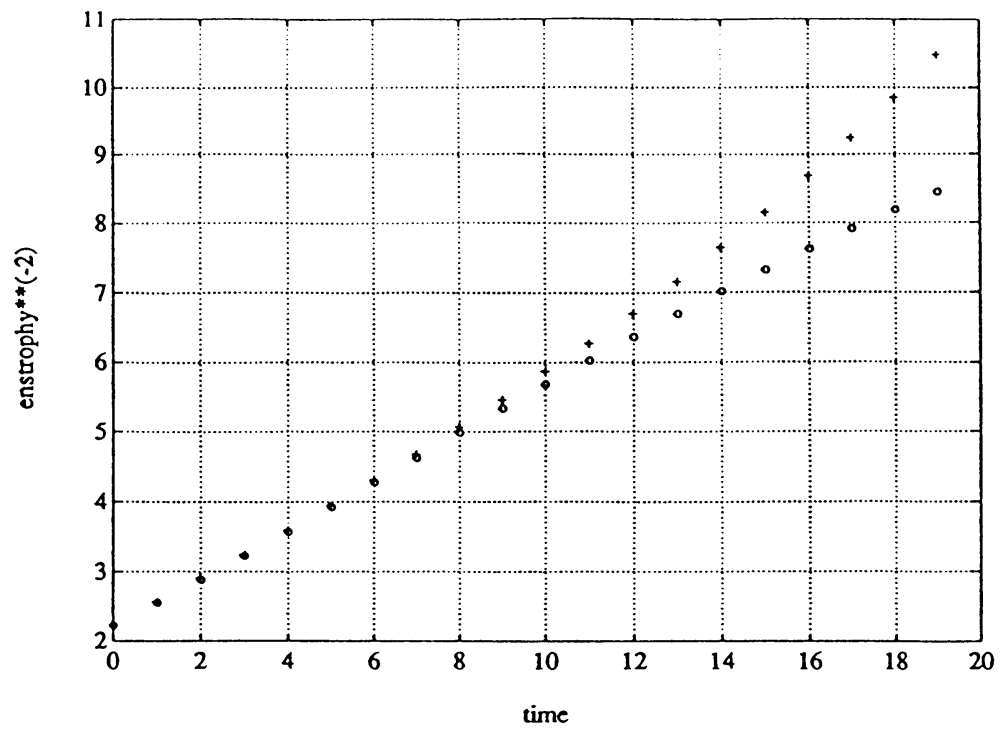

FIGURE 2

Comparison of (1.6) (+) and (4.1) (o) for $\nu=10^{-2}$

The reason is probably that, again owing to the radial symmetry of the vorticity, the particle paths are circular and a reordering $\Psi$ which follows these rotations will satisfy assumption $\mathrm{H} 2$.

In Figure 2 we have considered a much larger value of the viscosity $(\nu=.01$, the time step for the resolution of the diffusion had to be reduced to .1) to show the boundary effects on (4.1) as compared to (1.6). We see that (1.6) and (4.1) behave more or less equivalently, until the spreading of the vorticity reaches the boundary of the computational domain, which occurs very early for this value of $\nu$. Passed this time, the enstrophy computed corresponding to the scheme (4.1) begins decaying more slowly (and actually completely stops decaying after a while), while the decay increases with (1.6). This confirms that it is necessary to consider the diffusion scheme in the form (1.6) to obtain a decay property for all time. Also the speeding up of this decay can be interpreted by the fact that our estimates involve the $L_{1}$ norm of the vorticity. For a positive vorticity, for instance, this quantity is conserved by (1.6) until the vorticity reaches the boundary, and then decays. As for the behavior of (4.1), the saturation in the decay of the enstrophy can be easily explained by the fact that, since the vorticity in the present experiments is positive and the method (4.1), unlike (1.6), conserves the overall mass of the particles, we have

$$
\sum_{j} h^{2}\left|\omega_{j}\right|^{2} \geq K^{-1}\left(\sum_{j} h^{2} \omega_{j}(t)\right)^{2}=K^{-1}\left\|\omega_{0}\right\|_{1}^{2} .
$$


A practical conclusion is that for a given desired accuracy $\delta$, the estimate (1.7) can be used to determine the time $T^{*}$ passed which

$$
\left(\sum_{j} h^{2}\left|\omega_{j}(t)\right|^{2}\right)^{1 / 2}+\|\omega(t)\|_{L^{2}} \leq \delta ;
$$

then the size of the computational domain must be adjusted so that the vorticity is accurately approximated in $\left[0, T^{*}\right]$. Roughly speaking, we must have $K \simeq$ $\sqrt{\nu T^{*}} \simeq \delta^{-1}$, that is, the size of the computational domain is independent of the viscosity.

We finally discuss the case of a nonsmooth, nonradial vorticity. From the analysis in $\S 3$, it is quite clear that the rate of dissipation can exceed the one expected in the right-hand side of (1.7), if the vorticity is concentrated around high wave numbers, that is, if the vorticity is not smooth. In practice, a natural way to enforce a nonsmooth vorticity through the nonlinear part of the equation is to supplement (1.1)-(1.5) with a forcing term $f$ which is itself not smooth. As for the particle method, since the values of the weights are not affected by the convection, the mechanism for such an acceleration of the dissipation must involve the deformation of the Lagrangian mesh appearing in the diffusion formula (1.6). Actually, it can be observed that this deformation eventually leads to a local value of the viscosity which can exceed $\nu$ significantly (see [2] for a discussion of this point). We have performed experiments with smooth and nonsmooth forcing terms. To preserve a decay of the enstrophy like in (1.7), this forcing term must also decay in time. More precisely, it can be proved, for both the continuous equation and the particle method, that if $\|f(\cdot, t)\|_{L^{2}} \leq \alpha /\left(\nu t^{2}\right)$, then

$$
\|\omega(\cdot, t)\|_{L^{2}}^{2} \leq C /\left(\nu^{3 / 2} t\right),
$$

where the constant $C$ depends only on $\omega_{0}$ and $\alpha$. However, Figure 3 illustrates the fact that the rate of dissipation greatly depends on whether the forcing is smooth or not. We have chosen here two particular choices of $f$, with support in $\{|x| \leq 1\}$, the same $\mathbf{L}^{2}$ norm and mean values 0 , one piecewise constant, the other random:

$$
\begin{aligned}
& f_{1}(x)=.1 \frac{\operatorname{rand}(x)}{1+\nu t^{2}}, \\
& f_{2}(x)= \begin{cases}.005 / \sqrt{3}\left(1+\nu t^{2}\right) & \text { if }|x| \leq 1 / \sqrt{2}, \\
-.005 / \sqrt{3}\left(1+\nu t^{2}\right) & \text { if } 1 / \sqrt{2} \leq|x| \leq 1 .\end{cases}
\end{aligned}
$$

In the above definitions rand is a random generator in $[-.5,+.5]$. The viscosity has been set to $2 \times 10^{-3}$. The experiments were performed without reinitializations of particles, although the distribution is probably greatly distorted. This raises the question of whether the assumption $\mathrm{H} 2$ is necessary or only convenient for technical purposes in the proof. 


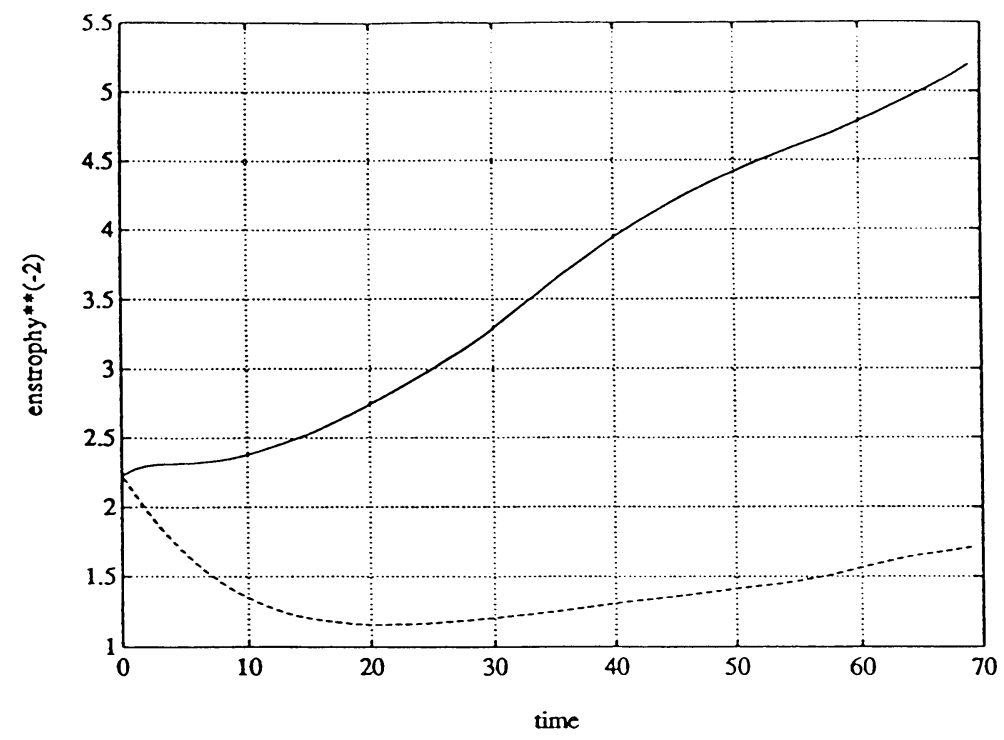

FIGURE 3

Evolution of the enstrophy for smooth (dotted line) and random forcing term

\section{ACKNOWLEDGMENT}

It is a pleasure for the author to thank E. Tadmor and H.-O. Kreiss for enlightening discussions at various stages of the present work.

\section{BIBLIOGRAPHY}

1. J. U. Brackbill and H. M. Ruppel, FLIP: a method for adaptively zoned particle-in-cell calculations of fluid flows in two dimensions, J. Comput. Phys. 65 (1986), 314-343.

2. G.-H. Cottet, A particle-grid superposition method for the Navier-Stokes equations, J. Comput. Phys. 89 (1990), 301-318.

3. G. H. Cottet and S. Mas-Gallic, Convergence of deterministic vortex methods for the NavierStokes equations (in preparation).

4. J. G. Heywood and R. Rannacher, Finite element approximation of the nonstationary Navier-Stokes problem, part 2: Stability of solutions and error estimates uniform in time, SIAM J. Numer. Anal. 23 (1986), 750-777.

5. S. Mas-Gallic, Thèse d'Etat, Université Paris 6, 1988.

6. M. E. Schoenbeck, $L^{2}$ decay for weak solutions of the Navier-Stokes equations, Arch. Rational Mech. Anal. 88 (1985), 209-222.

Department of Mathematics, University of California, Los Angeles, California 90024

Centre de Mathématiques Appliquées, CNRS, Ecole Polytechnique, 91128 Palaiseau Cedex, France 